\title{
ORIGINAL
}

\section{SERVICIOS SANITARIOS Y SOCIALES: NECESIDADES, PREFERENCIAS Y UTILIZACIÓN POR LOS ANCIANOS TRAS UN EPISODIO DE HOSPITALIZACIÓN *}

\section{José Antonio Iturria Sierra y Soledad Márquez Calderón.}

Unidad de Investigación en Servicios de Salud. Institut Valencià d’Estudis en Salut Pública.

(*) Este trabajo ha sido financiado con una ayuda de investigación del Institut Valencià d'Estudis en Salut Pública (proyecto 53/92).

\section{RESUMEN}

Fundamento: Muchos de los procesos que motivan un ingreso hospitalario en las personas ancianas pueden conllevar un deterioro de su nivel de salud en el momento del alta. Los objetivos de este trabajo son valorar la dependencia y necesidad de ayuda sociosanitaria que precisan las personas mayores de 64 años al ser dadas de alta en un hospital de agudos, la ayuda que los pacientes prefieren y la que realmente reciben al mes del alta.

Métodos: Se estudiaron 193 pacientes mayores de 64 años, ingresados en un hospital de Valencia entre febrero y abril de 1.994. Mediante una entrevista al alta y la historia clínica. se obtuvieron datos sobre caraclerísticas socio-demográficas, capacidad para los autocuidados. estado mental, diagnóstico principal y comorbilidad. Un equipo multidisciplinar valoró en cada caso la ayuda sociosanitaria requerida. Una segunda entrevista. un mes después del alta, aportó datos sobre la ayuda real recibida.

Resultados: En el momento del alta. el $17 \%$ de los pacientes precisaban ayuda parcial y el $21 \%$ ayuda completa. El $23 \%$ era candidato a recibir ayuda domiciliaria, el $9 \%$ a ser tratado en un hospital de día y el $6 \%$ en uno de crónicos. Los picientes demandaban mayoritariamente vivìr en su casa. Al mes del alta, sólo un $2 \%$ de los pacientes recibía ayuda domiciliaria, ninguno era tratado en hospital de día ni de crónicos, y un $3 \%$ volvía a estar ingresado en un hospital de agudos. Un $8 \%$ de los pacientes que vivían en su casa solos antes del ingreso y un $5 \%$ de los que vivían acompañados pasaron a vivir a casa de familiares.

Conclusiones: La realidad observada refkeja la falta de recursos sociosanitarios, situación que lieva en muchos casos a que la familia asuma los cuidados.

Palabras clave: Ancianos. Necesidades sociosanitarias. Alta hospitalaria.

Correspondencia:

José Antonio Iturria Sierra

Avenida de Cataluña 3. 510

Valencia-46010

\section{ABSTRACT \\ Health and Social Services: Needs, Preferences and their Use by the Elderly following Hospitalisation}

Background: Many processes behind the admission to hospital of elderly people can lead to a deterioration in their health at the time of discharge. The aims of the study are to assess the dependency on and need for socio-health care required by elderly people aged over 64 when discharged from a hospital for acute cases, the help that patients prefer and the help that they actually receive one month following their discharge.

Methods: A total of 193 patients aged over 64 and admitted to a hospital in Valencia between February and April 1994 were studied. Information on socio-demographic characteristics, selfcare capacity, mental state. main diagnosis and co-morbidity was obtained by means of an interview at the time of admission and the medical record. A multidisciplinary team evaluated the socio-health care required in each case. A second interview, one month after discharge from hospital, gathered data on the actual care received.

Results: At the time of admission, $17 \%$ of the patients needed partial care and $21 \%$ full care. $23 \%$ were candidates for receiving home help, $9 \%$ to be treated as out-patients and $6 \%$ in a chronic illness hospital. Most of the patients asked to live at home. One month after discharge from hospital. only $2 \%$ of patients were receiving home help, none were being treated as out-patients or in a chronic illness hospital and $3 \%$ hac once again admitted to a hospital for acute cases. $8 \%$ of the patients who were living at home alone before being admitted to hospital and $5 \%$ of those who werc living with someone else had gone to live with relatives.

Conclusions: The reality observed reflects the lack of socio-health resources. In many cases, this situation leads families to take on the care of the elderly themselves.

Key words: Flderly. Socio-health needs. Hospital discharge. 


\section{INTRODUCCIÓN}

Se estima que en 1996 el $14,5 \%$ de la población española tiene más de 64 años'. Este colectivo, por su importante morbilidad, es el que utiliza en mayor medida los recursos sanitarios. Por otra parte, sus necesidades de atención sociosanitaria se ven influidas por los cambios producidos en su soporte socio-familiar tradicional, con el consiguiente aumento del número de personas de esta edad que viven solas ${ }^{2-5}$.

El hecho de que la familia constituya la base de los cuidados informales y el soporte habitual del anciano, no significa que sea siempre el ámbito más idóneo para cubrir sus necesidades ${ }^{6.7}$. En un estudio realizado en Estados Unidos, el 68\% de los cuidadorcs eran igualmente ancianos, algo más de la mitad reconocían que los cuidados que prestaban eran insuficientes y el $98 \%$ decía que, por el hecho de serlo, se encontraba afectado su estilo de vida8.

En 1.991 el $16 \%$ de las personas de 65-74 años y el $21 \%$ de las mayores de 74 años, tuvieron un ingreso hospitalario ${ }^{9}$, porcentajes que tienden a aumentar en los últimos años ${ }^{10-11}$. En muchos casos, los procesos que motivan la estancia en el hospital pueden conllevar un deterioro de su nivel global de salud y un mayor grado de dependencia en el momento del alta.

Todos estos factores pueden dar lugar a que en los hospitales de agudos se produzcan demoras desde el alta médica al alta real de los pacientes ancianos, dado que estos centros asumirían en muchos casos funciones sociales. Estas circunstancias se ven agravadas cuando los recursos sociosanitarios alternativos son escasos o están poco coordinados entre sí.

No se han hallado estudios realizados en España que cuantifiquen la situación de los ancianos en el momento del alta, en lo referente a su nivel de salud y problemas de ubicación, paso que sería necesario para prever los recursos sociosanitarios adecuados.

Este estudio se realizó con los objetivos de: 1) valorar el grado de dependencia y el tipo de ayuda que necesitan las personas de 65 o más años dadas de alta en un hospital de agudos de la ciudad de Valencia, 2) describir la demanda expresada por los pacientes y por sus familiares en relación a su ubicación y cuidados, 3) conocer la ayuda real recibida tras el alta hospitalaria y compararla con las necesidades definidas previamente.

\section{MATERIAL Y MÉTODOS}

La población de referencia del estudio es la de las personas de 65 y más años de edad, residentes en el área sanitaria número 10 de Valencia y que fueron ingresadas en el Hospital General de dicha ciudad entre los meses de febrero y abril de 1994. El área 10 incluye una parte del casco antiguo de la ciudad de Valencia (con 177.811 habitantes, de los cuales el $20 \%$ son mayores de 64 años) y ocho municipios de la provincia (con 132.439 habitantes, de los cuales el $10 \%$ son mayores de 64 años).

Se eligieron 14 días del período de estudio (dos de cada día de la semana incluyendo domingos), en los que ingresaron en el hospital 252 personas mayores de 64 años. Se llevó a cabo un seguimiento de las mismas durante la hospitalización, con objeto de conocer el momento del alta con una antelación de 48-72 horas y realizar la entrevista. Se excluyeron 21 personas ( $8 \%$ ) que fallecieron durante las primeras horas tras el ingreso, $26(10 \%)$ que no pudieron ser entrevistadas por alta muy precoz o falta de notificación del alta y $12(4 \%)$ que no pudieron o no quisieron responder.

Antes del alta, se realizó una entrevista a los 193 pacientes que finalmente quedaron incluidos en el estudio (cuestionario disponible a petición a los autores), con objeto de obtener información sobre los siguientes aspectos: 
- Características socio-demográficas $(9$ preguntas).

- Apoyo socio-familiar (5 preguntas).

- Características de la vivienda (4 preguntas).

— Estado de salud, en términos de:

* Capacidad para los autocuidados, evaluada mediante el Indice de Actividades de la Vida Diaria de Katz ${ }^{12-1.3}$.

* Contincncia y movilidad (2 preguntas). Aunque el índice de Katz recoge información sobre estos dos aspectos, se completó la información con dos preguntas adicionales, dada la influencia de los mismos en la valoración de las necesidades de ayuda de los ancianos.

* Estado mental, valorado mediante el Short Portable Mental Status Questionnaire (SPMSQ) de Pfeiffer ${ }^{i 4}$.

- Preferencia sobre la ubicación del paciente tras el alta y demanda de servicios, expresadas por el paciente y por su familia (en los casos que estaba presente un familiar) (2 preguntas).

- Diagnóstico médico que motivó el ingreso del enfermo en el hospital y otras patologías que pudieran influir en su estado general de salud (comorbilidad).

- Además, los encuestadores anotaron tras cada respuesta, todo comentario que los pacientes hicieran referente a la misma.

Se constituyó un equipo multidisciplinar (EM) formado por un médico general, un rehabilitador, una enfermera y una trabajadora social. Este equipo se reunía semanalmente para estudiar los casos dados de alta, a partir de la información recogida en las entrevistas y los datos clínicos (historias clínicas). Se discutió cada caso con objeto de obtener una valoración consensuada de cuatro áreas fundamentales: nivel de dependen- cia psico-física, grado de apoyo social, caracterísicas de la vivienda e independencia económica.

La evaluación de la dependencia psico-física (expresada como nivel de necesidad de ayuda) se basó en datos de la capacidad para los autocuidados (índice de Katz), la continencia, la movilidad, el estado mental, las características de la enfermedad y el tratamiento aplicado; todo ello según el estado del enfermo el día del alta y sobre una previsión de la evolución a muy corto plazo y sin complicaciones. Se establecieron tres niveles de dependencia: autosuficiencia, necesidad de ayuda parcial y necesidad de ayuda completa. En estos dos últimos casos, se evaluó el tiempo diario de la avuda (períodos cortos y precisos, períodos largos o día y noche).

La valoración del nivel de apoyo socio-familiar, de la suficiencia de las condiciones de la vivienda y de la autonomía económi$\mathrm{ca}^{15}$, sirvieron de base (junto con la valoración del nivel de dependencia psico-física, la edad y las preferencias del paciente y su familia) para que el equipo multidisciplinar determinara la ubicación y atención considerada como más idónea para cada individuo, clasificada en una de las siguientes categorías:

* Domicilio habitual y red de asistencia primaria (para los ancianos calificados como autosuficientes).

* Ayuda domiciliaria (para personas que podían vivir en su hogar, pero que presentaban limitaciones en la autonomía y no tenían un apoyo socio-familiar suficiente).

* Hospital de día (para los ancianos que podían estar en su casa, pero seguían tratamientos de rehabilitación o padecían procesos crónicos leves y medios que requerían seguimiento).

* Hospital de agudos (para procesos curativos y cuidados paliativos de corta estancia),cuando se consensuó que el alta era inapropiada. 
* Residencias (para personas con limitaciones de carácter permanente que les impedían vivir solos y sin suficiencia social, familiar o económica).

* Hospital de crónicos o centros sociosanitarios (para convalecientes en rehabilitación, pluripatologías, cuidados paliativos y largas estancias especiales - como demencias-).

A los 30 días tras el alta hospitalaria se estableció contacto telefónico con cada paciente para comprobar la ubicación real del mismo y el tipo de ayuda o servicio que recibía.

Los resultados se presentan de forma descriptiva, expresando las frecuencias en porcentajes. Los datos fueron analizados con el programa Epiinfo.

\section{RESULTADOS}

\section{Características sociodemográficas}

De los 193 sujetos del estudio, $106(55 \%)$ eran varones y 87 (45\%) mujeres. La edad media de los hombres era de 73,6 años (DE 6,6) y la de las mujeres de 76,5 años (DE 7,2 ). En cuanto al nivel educativo, un $21 \%$ de los pacientes eran analfabetos, un $60 \%$ leían y escribían, un $17 \%$ habían concluido los estudios primarios y un $2 \%$ tenían estu- dios medios o universitarios. Las ocupaciones más frecuentes antes de la jubilación eran las de trabajador no cualificado $(38 \%)$ y ama de casa (36\%). El $69 \%$ vivían en su casa con su cónyuge u otro familiar, el $13 \%$ en casa de familiares, otro $13 \%$ solos y un $5 \%$ en residencias ${ }^{15}$.

\section{Necesidad de ayuda determinada por el equipo multidisciplinar}

La valoración de la ayuda necesaria realizada por el equipo multidisciplinar (EM) se basó en los resultados de los índices de salud recogidos en la encuesta, los datos clínicos (diagnóstico principal y comorbilidad), el apoyo socio-familiar, las características de la vivienda y la independencia económica (todos estos datos básicos son objeto de otra publicación ${ }^{15}$ ), con los siguientes resultados: 120 pacientes $(62 \%)$ eran autosuficientes en el momento del alta, $33(17 \%)$ precisaban ayuda parcial y $40(21 \%)$ ayuda completa. De los pacientes que requerían algún tipo de ayuda, $45(62 \%)$ la necesitaban en períodos cortos y precisos del día, 11 (15\%) en períodos largos, y $17(23 \%)$ durante día y noche.

\section{Preferencias del paciente y su familia sobre la ubicación y atención}

En la tabla 1 se presentan los resultados sobre las preferencias expresadas por los pa-

Tabla 1

Preferencias de los pacientes y familiares en relación a la ubicación del enfermo tras el alta

\begin{tabular}{|c|c|c|c|c|}
\hline & \multicolumn{4}{|c|}{ Preferencia de ubicación tras el alta } \\
\hline & \multicolumn{2}{|c|}{ Del paciente } & \multicolumn{2}{|c|}{ De la familia } \\
\hline & $N$ & $(\%)$ & $N$ & $(\%)$ \\
\hline Su casa & 112 & $(70.0)$ & 62 & (60) \\
\hline Su casa con ayuda domiciliaria & 31 & $(19,5)$ & 26 & (25) \\
\hline Casa de familiares & 13 & $(8.0)$ & 11 & (11) \\
\hline Residencia & 4 & $(2,5)$ & 3 & (3) \\
\hline Hospital & 0 & $(0)$ & 1 & (1) \\
\hline No contesta & 33 & & 90 & \\
\hline Total & 193 & $(100)$ & 193 & $(100)$ \\
\hline
\end{tabular}


cientes y sus familiares con relación a la ubicación después del alta. De los 160 pacientes que respondieron a esta cuestión, la mayor parte $(89 \%)$ querían vivir en su casa, si bien algunos de éstos manifestaban necesitar ayuda domiciliaria. Sólo 4 pacientes $(2.5 \%)$ pedían ir a una residencia. En los 103 casos que pudo entrevistarse a un familiar acompañante, el $85 \%$ deseaba que el paciente fuera a vivir a su propio domicilio y un $11 \%$ pensaba que el paciente debía vivir en casa de familiares. En la mayor parte de los casos (69 de los $72 \mathrm{cn}$ que se disponía de la demanda expresada tanto por el paciente como por un familiar), las preferencias de ambos coincidían.

\section{Ubicación más adecuada según el equipo multidisciplinar y situación real al mes del alta}

El EM determinó por consenso la ubicación y atención más adecuada para cada paciente (tabla 2). Un $43 \%$ de los pacientes (83 personas) podían remitirse a su casa sin ayuda y ser atendidos por la red de asistencia primaria. Un 22\% (43 pacientes) podrían ser remitidos a su casa si pudieran recibir ayuda domiciliaria social y/o médica, y un $9 \%$ más (18 pacientes) si se dispusiera de hospitales de día. El 19\% (37 pacientes) requería una plaza en una residencia y el $6 \%$ (12 pacientes) en un hospital de crónicos.

Un mes después del alta hospitalaria, el $62 \%$ de los ancianos vivían en su domicilio con familiares, el $10 \%$ vivían solos y el $13 \%$ en casa de otros familiares (Tabla 2). La ubicación aconsejada por el EM y la real constatada a los 30 días del alta coincidía en un $92 \%$ de los pacientes cuya ubicación adecuada era el propio domicilio sin necesidad de ayuda, encontrándose un $6 \%$ de ellos en casa de familiares $(6 \%)$. De los pacientes cuya ubicación idónea era su casa con ayuda domiciliaria, sólo uno ( $2 \%$ ) la estaba recibiendo al mes del alta. La mayoría de las personas de este grupo ( $83 \%$ ) estaban en su casa sin ayuda y un 5\% volvía a estar ingresado en un hospital de agudos. Ninguno de los 18 pacientes en que el EM recomendaba ser tratado en un hospital de día recibía este tipo de atención. De éstos, el $78 \%$ estaba en su domicilio sin ayuda, el $11 \%$ en casa de familiares y el $5 \%$ en una residencia. Tampoco ninguno de los pacientes en que se recomendaba el ingreso en un hospital de crónicos recibía esta atención. La situación más frecuente en este grupo (42\% de los pacientes) era residir en casa de familiares. De los ancianos que precisaban una plaza

Tabla 2

Ubicación adecuada según el equipo multidisciplinar y ubicación real de los pacientes tras el alta hospitalaria

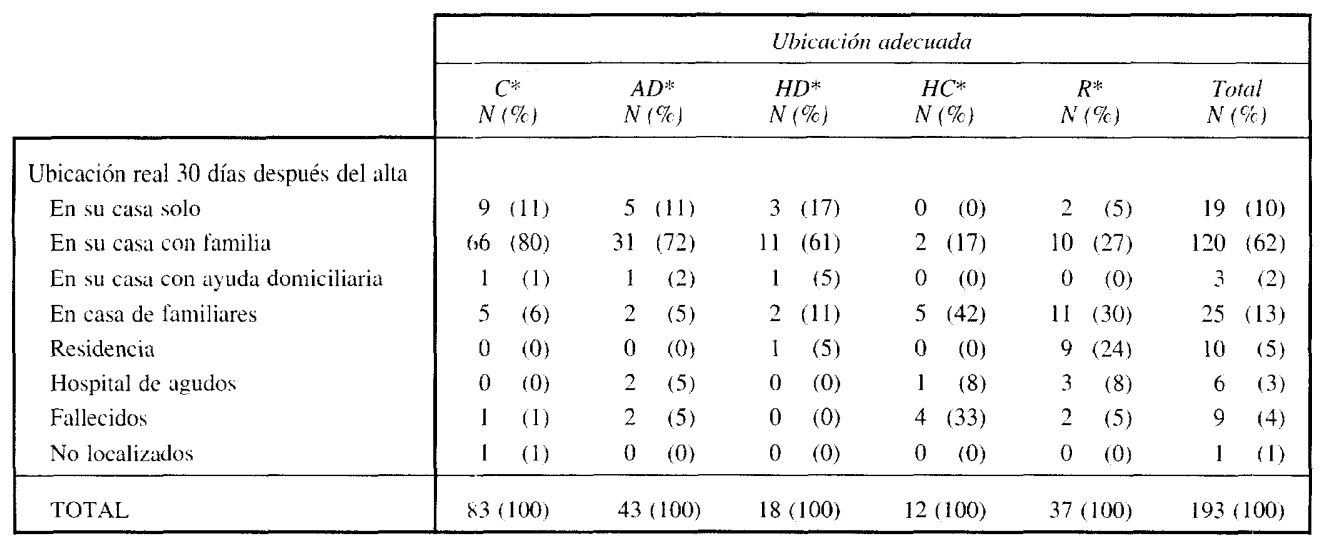

* C: En su casa sin ayuda. AD: Ayuda domiciliaria. HD: Hospital de día. HC: Hospital de crónicos. R: Residencia. 
en residencia, el $24 \%$ la tenía al mes del alta, el $30 \%$ vivía en casa de familiares y el $32 \%$ en su hogar sin ayuda domiciliaria (tabla 2).

\section{Nivel de dependencia y ubicación adecuada según la edad}

La edad se mostró una variable muy correlacionada con el nivel de dependencia y, consecuentemente, con la ubicación considerada como más adecuada por el EM después del alta (tabla 3).

En el momento del alta, el $77 \%$ de los pacientes menores de 70 años eran autosuficientes, frente a un $71 \%$ de los del grupo de 70-79 años y sólo un 33\% de los mayores de 79 años. El $41 \%$ de las personas de este último grupo de edad presentaba una dependencia total el día del alta.

En el grupo de los pacientes mayores de 79 años, sólo el $13 \%$ podía ir a vivir a su casa sin ayuda tras el alta, en un $48 \%$ de ellos el EM valoró como necesaria el alta a una residencia y en un $11 \%$ a un hospital de crónicos. La proporción de pacientes en los que se valoró que la ubicación adecuada era una residencia u hospital de crónicos fue mucho más baja en el grupo de 70-79 años ( $12 \%$ y $6 \%$ respectivamente) y en el de menores de 70 años ( $2 \%$ y 0 respectivamente) (tabla 3).

\section{Ubicación adecuada y real según el domicilio anterior al ingreso hospitalario}

De las 26 personas que vivian solas en su domicilio antes del ingreso, el EM valoró que $18(69 \%)$ podían seguir viviendo en su casa, aunque 5 casos precisarían ayuda domiciliaría y 4 casos la atención en un hospital de día. Al mes del alta, un $84 \%$ seguía en su domicilio sin recibir ayuda domiciliaria, un $8 \%$ en una residencia y un $8 \%$ habían pasado a vivir a casa de familiares (tabla 4).

La situación más frecuente antes del alta era vivir en el propio domicilio con otros familiares (132 personas). El EM valoró que el $85 \%$ de estas personas (113) podían continuar viviendo en su casa tras el alta, aunque era frecuente la necesidad de ayuda domiciliaria y la atención en hospital de día. Al mes del alta, el $91 \%$ de estas personas vivían en su domicilio, aunque sólo 2 de ellas recibían ayuda domiciliaria. Las siguientes situaciones más frecuentes en este grupo fueron el traslado a casa de un familiar $(5 \%)$ y estar

Tabla 3

\begin{tabular}{|l} 
Dependencia y ubicación adecuada según la edad \\
\cline { 3 - 7 } \\
\cline { 3 - 7 }
\end{tabular}

* Valoración realizada por el equipo multidisciplinar. 
Tabla 4

Ubicación adecuada y real según el domicilio anterior al ingreso hospitalario

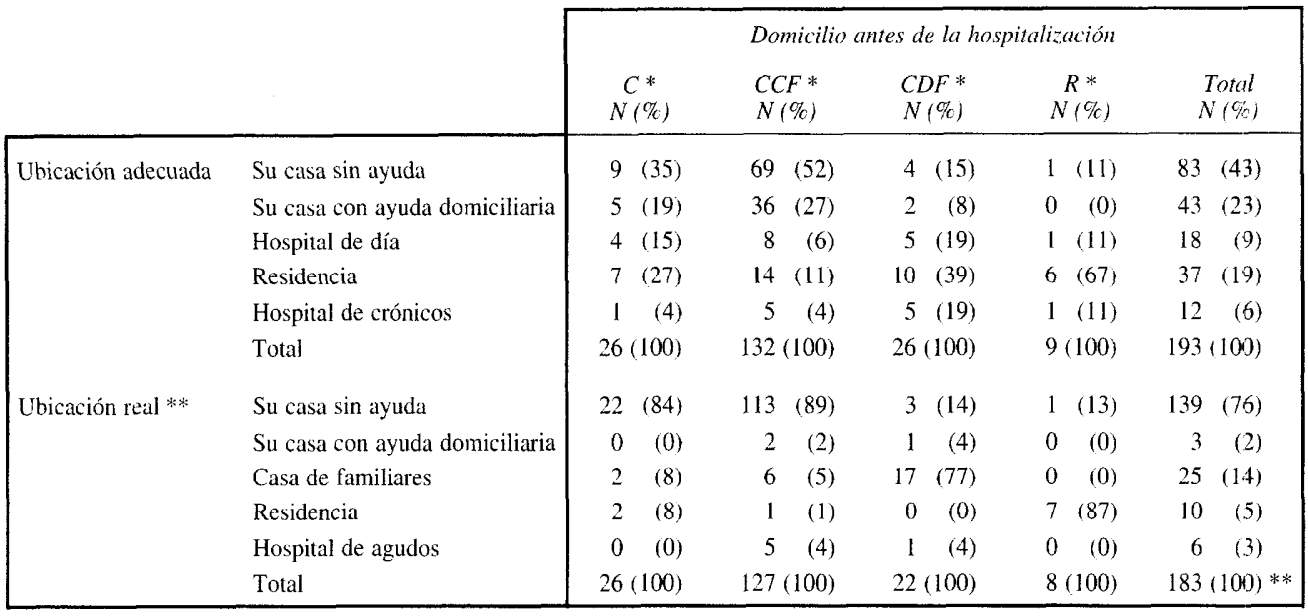

* C: Su casa solo. sin ayuda. CCF: Su casa con familiti. CDF: En casa de familiares. R: Residencia.

** Se excluyen 9 fallecidos y una persona no localizada.

ingresado de nuevo en un hospital de agudos $(4 \%)$ (tabla 4$)$.

En 10 de las 26 personas $(39 \%)$ que $v i$ vían en casa de familiares antes del ingreso, el EM determinó que la ubicación adecuada era una residencia. Al mes del alta, 17 personas $(77 \%)$ continuaban en casa de familiares y $4(18 \%)$ habían pasado a vivir a su propio domicilio (tabla 4 ).

Nueve personas vivían en una residencia antes del ingreso. La mayoría (87\%) continuaban en ella un mes después del alta (tabla 4).

\section{Concordancia entre las preferencias de los pacientes y la ubicación adecuada y real tras el alta}

De los 112 pacientes que expresaban el deseo de volver a su domicilio (sin demandar ayuda), el EM valoró que el propio domicilio era la ubicación adecuada en el $90 \%$ de los casos, si bien 25 de estos pacientes requerían ayuda domiciliaria. En 9 personas (8\%) el EM determinó que la ubicación adecuada era una residencia. Al mes del alta, el
$86 \%$ de estos pacientes vivía en su propio domicilio, sólo uno recibía ayuda domiciliaria y un 3\% estaba en una residencia (tabla 5).

En el $32 \%$ de los casos que demandaban ir a su casa con ayuda domiciliaria el EM coincidía en la valoración, y en otro $32 \%$ el equipo determinó que la ubicación adecuada era una residencia. Al mes del alta hospitalaria, sólo 2 pacientes $(6 \%)$ recibían ayuda domiciliaria y $1(3 \%)$ estaba en una residencia (tabla 5).

En 8 de los 13 pacientes que expresaban el deseo de vivir en casa de familiares, el EM valoró que podían vivir en su propio domicilio, si bien algunos de ellos requerían ayuda domiciliaria o ser atendidos en un hospital de día. La situación más frecuente de estos pacientes al mes del alta era vivir en casa de familiares (53\%), seguido del propio domicilio sin ayuda (31\%) (tabla 5).

\section{DISCUSIÓN}

Una alta proporción de los sujetos del estudio necesilaba ayuda parcial o completa, 
Tabla 5

Concordancia entre la demanda del paciente y las ubicaciones adecuada y real tras el alta hospitalaria

\begin{tabular}{|c|c|c|c|c|c|c|}
\hline & & \multicolumn{5}{|c|}{ Demanda del paciente } \\
\hline & & $\begin{array}{c}C * \\
N(\%)\end{array}$ & $\begin{array}{c}A D^{*} \\
N(\%)\end{array}$ & $\begin{array}{l}C D F \\
N(\%)\end{array}$ & $\begin{array}{c}R * \\
N(\%)\end{array}$ & $\begin{array}{c}\text { Total } \\
N(\%)\end{array}$ \\
\hline \multirow[t]{7}{*}{ Ubicación adecuada } & En casa & $69(62)$ & $5(16)$ & $4 \quad(31)$ & $0 \quad(0)$ & $78 \quad(49)$ \\
\hline & Ayuda domiciliaria & $25 \quad(22)$ & $10 \quad(32)$ & $2(15)$ & $0 \quad(0)$ & $37 \quad(23)$ \\
\hline & Hospital de día & $7 \quad(6)$ & $6 \quad(20)$ & $2(15)$ & $0 \quad(0)$ & $15 \quad(9)$ \\
\hline & Hospital de agudos & $1 \quad(1)$ & o $\quad(0)$ & $0 \quad(0)$ & $0 \quad(0)$ & 1 (I) \\
\hline & Hospital de crónicos & $1 \quad(1)$ & $0 \quad(0)$ & I (8) & () $\quad(0)$ & 2 (1) \\
\hline & Residencia & $9 \quad(8)$ & $10 \quad(32)$ & $4(31)$ & $4(100)$ & $27 \quad(17)$ \\
\hline & Total & $112(100)$ & $31(100)$ & $13(100)$ & $4(100)$ & $160(100)$ \\
\hline \multirow[t]{9}{*}{ Ubicación real } & Fn casa solo & 13 (11) & $4(13)$ & $0 \quad(0)$ & $0 \quad(0)$ & $17(10)$ \\
\hline & En casa con familiares & $83 \quad(74)$ & $19(61)$ & $4(31)$ & $0 \quad(0)$ & $106 \quad(66)$ \\
\hline & Ayuda domiciliaria & $1 \quad(1)$ & $2 \quad(6)$ & $0 \quad(0)$ & $0 \quad(0)$ & $3 \quad(2)$ \\
\hline & Casa de familiares & $6 \quad(5)$ & 4. (13) & $7(53)$ & 1 (25) & $18(11)$ \\
\hline & Hospital de agudos & $2 \quad(2)$ & 1 (3) & $1 \quad(8)$ & $\begin{array}{ll}0 & (0)\end{array}$ & $4 \quad(3)$ \\
\hline & Residencia & $3 \quad(3)$ & $1 \quad(3)$ & $1 \quad(8)$ & $3(75)$ & $8 \quad(5)$ \\
\hline & Fallecidos & $3 \quad(3)$ & $\begin{array}{ll}0 & (0)\end{array}$ & $0 \quad(0)$ & $\begin{array}{ll}0 & (0)\end{array}$ & $3 \quad(2)$ \\
\hline & No localizados & 1 (1) & $0 \quad(0)$ & $0 \quad(0)$ & $0 \quad(0)$ & 1 (1) \\
\hline & Total & $(100)$ & $31(100)$ & $13(100)$ & $4(100)$ & $160(100)$ \\
\hline
\end{tabular}

* C: En su casa. AD: En casa con ayuda doniciliaria, CDF: En casa de familiares. R Residencia.

especialmente los mayores de 79 años; y la realidad observada al mes del alta refleja la falta de recursos sociosanitarios alternativos y/o su falta de utilización, situación que lleva a que muchos pacientes dependientes vivan en su casa sin ningún tipo de ayuda y a que la familia se haga cargo de los cuidados de un alto porcentaje de ancianos.

El estudio presenta algunas limitaciones, sobre todo relacionadas con la subjetividad del método de valoración por consenso del equipo multidisciplinar ${ }^{15}$. Así, cuando se determinó la ubicación más adecuada del paciente tras el alta, se excluyó explícitamente el domicilio de un familiar como alternativa. Esta exclusión se basaba en dos razones: por una parte, la demanda más frecuente de los interesados y sus familiares era la de vivir en su propio hogar; por otra, si los recursos sociosanitarios disponibles en la sociedad fueran suficientes, probablemente sería mucho menos frecuente la necesidad de que los familiares trasladaran al anciano a su propia casa, con la sobrecarga de trabajo y pérdida de calidad de vida en los cuidadores que suponen este tipo de situaciones $^{8}$. Sin embargo, aunque no se ha contemplado esta posibilidad, no cabe duda que en bastantes casos (aún existiendo recursos alternativos) los familiares de los ancianos seguirían prefiriendo cuidarlos ellos mismos.

La determinación del tipo de recurso que precisaba cada individuo según el grado de necesidad se veía dificultada, en algunos casos, por la falta de separación clara entre algunas alternativas. Así, a veces resulta difícil en enfermos crónicos con pluripatología, delimitar las indicaciones de residencia asistida y hospital de crónicos. En estos casos se siguió un criterio clínico, con la decisión de que las personas que precisaban mayor cantidad y calidad de cuidados irían a un centro de crónicos y las que mantenían cierto grado de autonomía podrían ir a una residencia asistida. Lo mismo ocurría en enfermos con dependencias parciales en el momento de dictaminar sobre su ubicación en residen- 
cias de válidos o en su casa con ayuda domiciliaria, prefiriéndose las primeras si había apoyo socio-familiar o vivienda insuficientes o nivel económico precario y sin posibilidad de ayuda.

Por otra parte, al decidir sobre el tipo de atención sociosanitaria adecuada a cada paciente tras el alta, no se tuvieron en cuenta sólo los recursos que están al alcance de los ancianos que viven en el área 10 de Valencia, sino el total de las posibilidades de medios existentes, estuvieran o no presentes en nuestra Comunidad. Esto responde a que el objetivo del estudio no era la distribución de las ayudas disponibles, sino la estimación de las necesidades de los ancianos.

La demanda expresada, bien sea por el paciente o por sus familiares ha estado determinada, no sólo por la necesidad percibida de servicios, sino también por el desconocimiento de las posibilidades de medios existentes. Cada encuestado contestó según su criterio, ya que la pregunta se formuló de forma abierta. De esta forma se evitó la sobredemanda de ayuda, a la vez que se respetaba el criterio de no crear expectativas que luego no podían satisfacerse por falta del recurso ofertado.

E1 21\% de los pacientes precisaban ayuda completa en el momento del alta. debido a su nivel de dependencia psico-física. Este porcentaje es similar al hallado en otros estudios realizados en España y otros paí$\operatorname{ses}^{3.4 .16}$.

Según la valoración del equipo multidisciplinar, el $75 \%$ de los pacientes podía vivir en su domicilio tras el alta, si bien en muchos de ellos estaba condicionado a recibir ayuda domiciliaria o atención en un hospital de día. Al mes del alta, el $72 \%$ de los pacientes vivían en su casa y el $13 \%$ en casa de familiares, pero sólo 3 pacientes recibían ayuda domiciliaria y ninguno era atendido en un hospital de día. Tampoco ninguno de los pacientes en que el equipo multidisciplinar recomendaba el ingreso en un hospital de crónicos recibía este tipo de atención. $\mathrm{Al}$ mes del alta, la mayor parte de los ancianos continuaban viviendo en el mismo lugar que antes del ingreso; pero cabe destacar que un $8 \%$ de las personas que vivían solas antes del ingreso habían pasado a una residencia y un $8 \%$ a casa de familiares, el $5 \%$ de las que vivían en su propia casa con familia habían pasado también al domicilio de familiares, y alrededor de un 3\% del total de pacientes volvían a estar ingresados en un hospital de agudos. El porcentaje de personas que cambiaban de lugar de residencia era muy inferior al $40 \%$ descrito por Morrow-Howell et $\mathrm{al}^{17}$, pero en este estudio los pacientes eran un grupo ya seleccionado para planificación del alta (probablemente con mayores necesidades) y en el contexto social de Estados Unidos. La ubicación inadecuada de los pacientes tras el alta puede cifrarse en torno al $28 \%$, cifra similar a la citada por Masdeu et $\mathrm{al}^{18}$.

Todos estos datos indican que, en la mayor parte de los casos, es la familia quien se hace cargo de prestar los cuidados precisos cuando los recursos públicos son escasos o inexistentes, con el previsible impacto en la calidad de vida de los cuidadore ${ }^{19-20}$. La demanda expresada por los pacientes y familiares era mayoritariamente la de ir a vivir al propio domicilio. Probablemente, el desconocimiento de la existencia de recursos como la ayuda domiciliaria y los hospitales de crónicos motivaba que éstos no se solicitaran.

Entre las posibles causas de la no satisfacción de las necesidades, está sin duda la falta de recursos alternativos al hospital de agu$\operatorname{dos}^{21}$; en este caso concreto no se disponía de hospitales de día y había pocas plazas de crónicos para toda la ciudad de Valencia. Sin embargo, la literatura señala algunas causas de demora en el alta, que podrían relacionarse también con la falta de atención adecuada tras un episodio de hospitalización. Entre ellas están la falta de coordinación entre los distintos servicios sociosanitarios 22 y la planificación inadecuada del alta ${ }^{23}$. Es posible, aún en un contexto de recursos escasos, que 
los programas de planificación del alta puedan mejorar la satisfacción de las necesidades de atención de los ancianos, mediante su identificación precoz y la búsqueda - desde el inicio del ingreso-_- del recurso necesario, sea éste una plaza en residencia, en hospital de crónicos o la atención domiciliaria.

Los programas de planificación del alta, ampliamente difundidos en Estados Unidos, se plantearon con el objetivo de reducir las estancias en hospitales de agudos por motivos no médicos, aunque también van dirigidos a mejorar la calidad de vida de los an$\operatorname{cianos}^{24}$. Aunque las distintas evaluaciones realizadas muestran resultados controvertidos en relación a la disminución de estancias $^{25-29}$, parece que son efectivos en disminuir la tasa de reingresos y aumentar la satisfacción de los pacientes ${ }^{25.29-30}$. También los programas de ayuda especializada en el domicilio han conseguido disminuir los reingresos y aumentar la tasa de permanencia en la casa a las seis semanas tras el alta ${ }^{31}$.

Aunque no se pretende que los resultados de este trabajo sean extrapolables a otros contextos, presentan una aproximación a la necesidad de recursos alternativos a los hospitales de agudos. El envejecimiento progresivo de la población, con un aumento del número de ancianos y en especial, de los mayores de 80 años -con mayor nivel de dependencia - conviertc a este grupo cn una fuente creciente y segura de demanda de estos recursos sociosanitarios y de su adecuada gestión y coordinación ${ }^{1,32}$.

\section{AGRADECIMIENTOS}

A Carmen Illa, Enriqueta Llopis y Fernando Blasco por su participación como equipo multidisciplinar en la valoración de los ancianos. A la Dirección Médica y al equipo de trabajadores sociales del Hospital General de Valencia, que han permitido el acceso a los diferentes servicios hospitalarios.

\section{BIBLIOGRAFÍA}

1. Instituto Nacional de Estadística. Proyección de la Población Española para el Período 1980-2010. Tomo para el conjunto nacional. Madrid: I.N.E.; 1987.

2. Garay J, Gracia J. La enfermería geriátrica en el año 2000. Geriátrika 1990; 6:75-7.

3. Jeangros C, Dubois-Arber F, Paccaud F. Soins ambulatoires aux personnes âgées dans le canton de Genève: que font les médecins praticiens? Méd Hyg 1989; 47:3418-24.

4. Jones EJ, Densen PM, Brown SD. Posthospital needs of elderly people at home: findings from an eigth-month follow-up study. Health Serv Res $1989 ; 24: 643-64$.

5. Gómez A, Varona N, Alonso MC, García L, Menéndez J. Salud percibida, utilización de servicios y consumo de medicamentos en población anciana no institucionalizada. Aten Primaria 1993: 11:233 8.

6. Hall JA, Epstein AM, McNeil BJ. Multidimensionality of health status in an elderly population. Med Care 1989:27 (3 Supl): 168-77.

7. Counte MA, Glandon GL. A panel study of life stress, social support, and the health services utilization of older persons. Med Care 1991: 29:34861.

8. Lafata JE, Koch GG, Weissert WG. Estimating activity limitation in the noninstitutionalized population: a method for small areas. Am J Public Health $1994 ; 84: 1813-9$.

9. Instituto Nacional de Estadística. Encuesta de Morbilidad Hospitalaria 1991. Madrid: I.N.E.; 1994.

10. Compañ L, Pérez-Hoyos S, García AM. Utilización hospitalaria en ancianos en la Comunidad Valenciana. Rev Geront 1994; 3:168-72.

11. Compañ L, Portella E. Cambios en la utilización hospitalaria de la población anciana en la década de los 80: el caso de las cataratas. Rev Geront 1995; 1:23-8.

12. González JI, Rodríguez C, Diestro P, Casado MT, Vallejo MI, Calvo MJ. Valoración funcional: comparación de la escala de Cruz Roja con el índice de Katz. Rev Esp Geriat Geront 1991; 26:197-202.

13. Rowland D. Measuring the elderly's need for home care. Health Aff 1989; Winter:39-49.

14. Kane RA, Kane RL. Assessing the elderly. 40 ed. Lexington: LexingtonBooks; 1981. 
15. Iturria JA. Márquez S. Situación de los ancianos tras un episodio de hospitalización: estado de sálud, recursos materiales y apoyo scicial. Rev Geront 1996; 6:92-7.

16. González JI, Pérez J. Rodríguez I, Salgado A, Guillén F. Geriatría y asistencia geriátrica: para quién y cómo (II). Med Clin (Barc) 1991; 96:2228.

17. Morrow-Howell N, Proctor E. Discharge destinations of Medicare patiens receiving discharge planning: who goes where? Med Care 1994; 32:486-97.

18. Masdeu M, Pérez MF. Servicio de atención domiciliaria. Todo Hosp 1992; 84:43-5.

19. Kemper $\mathbf{P}$. The use of formal and informal home care by the disabled elderly. Health Serv Res 1992: 27:421-51.

20. Abraham PY, Berry ML. Necds of the family caregivers of frail elderly. Can J Public Health 1992: 83:147-9.

21. Toseland RW. Newman ES. Admission proccdure in skilled nursing facilities. J Long Term Care Adm 1984; 12:3-9.

22. Barker WH, Williams TF, Zimmer JG et al. Geriatric consultation teams in acute hospital: impact on back-up of elderly patients. J Am Geriatric Soc $1985 ; 33: 422-8$.

23. Restuccia JD, Holloway DC. Barriers to apropiate utilization of an acute care facility. Med Care $1976 ; 14: 559-73$.
24. Manheim LM, Guihan M, Hughes SL. Organizational characteristics, client characteristics and the delivery of home care services. Med Care Res \& Rev 1995; 52:517.

25. Haddock KS. Collaborative discharge planning: nursing and social services. Clin Nurse Spec 1994: 8:248-52.

26. Boone CR, Coulton CJ, Keller SM. Impact of early social work services on lenght of stay. Soc Work Health Care 1981; 7:1.

27. Cohen M, Tell E, Wallacks S. Client related risk factors of nursing home entry among elderly. J Gerontol 1986; 41:785.

28. Mozes B, Halkin H, Katz A, Shiff E. Reduction of redundant hospital stay through controlled intervention. Lancet 1987; i:968-9.

29. Evans RL, Hendricks RD. Evaluating hospital discharge planning: a randomized clinical trial. Med Care 1993; 31:358-70.

30. Naylor M, Brooten D, Jones R et al. Comprehensive discharge for the hospitalized elderly. A randomized clinical trial. Ann Intern Med 1994: 120:999-1006.

31. Martin F, Oyewole A, Moloney A. A randomized controlled trial of a high support hospital discharge team for elderly people. Age Ageing 1994; 23:228-34.

32. Zedlewski SR, McBride TD. The changing profile of the elderly: effects on future long-term care needs and financing. Milbank Q 1992; 70:247-75. 